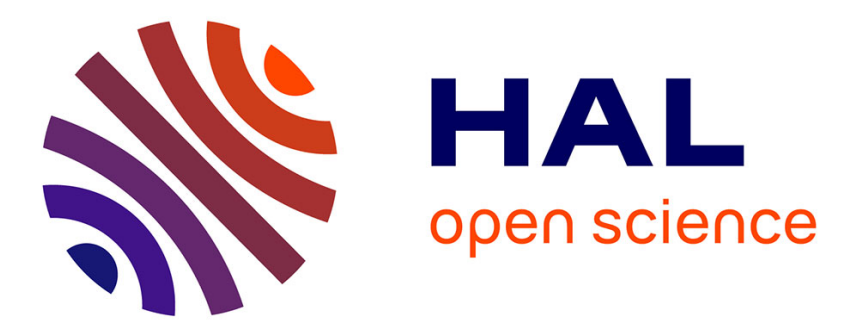

\title{
Resources management and decision support in a system of information systems
}

Majd Saleh, Marie-Helene Abel

\section{To cite this version:}

Majd Saleh, Marie-Helene Abel. Resources management and decision support in a system of information systems. 2016 International Conference on Industrial Informatics and Computer Systems (CIICS), Mar 2016, Dubai, United Arab Emirates. pp.1-5, 10.1109/ICCSII.2016.7462434 . hal-01396295

\section{HAL Id: hal-01396295 \\ https://hal.science/hal-01396295}

Submitted on 25 Nov 2016

HAL is a multi-disciplinary open access archive for the deposit and dissemination of scientific research documents, whether they are published or not. The documents may come from teaching and research institutions in France or abroad, or from public or private research centers.
L'archive ouverte pluridisciplinaire HAL, est destinée au dépôt et à la diffusion de documents scientifiques de niveau recherche, publiés ou non, émanant des établissements d'enseignement et de recherche français ou étrangers, des laboratoires publics ou privés. 


\title{
Resources Management and Decision Support in a System of Information Systems
}

\author{
Majd Saleh, Marie-Hélène Abel \\ Sorbonne Universités, Université de \\ Technologie de Compiègne, CNRS, \\ UMR 7253 Heudiasyc \\ Compiègne, France \\ Email: \{majd.saleh,marie-helene.abel $\} @$ utc.fr
}

\begin{abstract}
Recently, new Information Systems are being introduced to users on regular basis. At a certain point, users might deal with several systems producing overwhelming amount of information and resources. This is challenging on many levels, as the user has to look for information and resources in different Information Systems. One of the most important challenges here is the way to manage the resources produced from those systems. Another important one is to produce knowledge from these resources and provide support for decision making process. This also applies to complex systems such as System of Information Systems (SoIS). Thus, we aim to investigate resources management and decision support in a System of Information Systems (SoIS). We plan to address this issue by introducing an architectural model of SoIS. This model will provide guidance to build a first prototype. The SoIS operates as a single entry point for several Information Systems granting the user access to information and resources produced from multiple Information Systems. It also provide the ability to track users' activities to create an added value not possible to maintain when those systems were operating separately. Furthermore, this encompassing solution is possible by introducing a knowledge base as a leader system to the orchestration of SoIS. This knowledge base can take the role for storing, organizing, and sharing resources from different Information Systems. Thus, provide the link between resources present in different systems and facilitate resources management and decision making in a System of Information Systems.
\end{abstract}

\section{INTRODUCTION}

Information Systems are turning to be more complex, and changing from easy-to-use to hard-to-use. However, effectiveness and efficiency in information systems is not satisfying [1]. This universal nature of existing Information Systems has generated a strong interest in using an existing set of systems as the basis for a System of Systems [2], as these systems need to learn how to work and communicate with each other in order to gather information and resources and produce new ones emerged from existing systems. Therefore, securing a competitive advantage does no longer rely only on efficiency, quality, and customer responsiveness. While each of these factors is important, the ability to deploy available solution to emerging problems by aggregating existing Information Systems and providing ease of access to the information and resources in these systems is of great importance. This makes innovation, flexibility, coordination, integration, and speed of retrieving and managing resources the new success factors of today's work environment. Those factors can be achieved through the concept of System of Information Systems (SoIS).
The desired SoIS needs to connect Information Systems that cross organizational boundaries, come from multiple domains, and generates an overwhelming amount of information and resources. Users struggle to deal with the information and resources produced by each Information System independently by traversing through these systems and keeping track of the generated information and resources separately. Additionally, users cannot possibly infer new knowledge from those resources as they are residing in different systems and environments. The new inferred knowledge can be in the form of users' activities around those resources, which will help determining users' competency in a certain subject by their activities around that subject. A solution might be found in a well-established architectural model of System of Information Systems that provides guidance to produce such solution. The construction of SoIS, however, requires long-term projects that involve comprehensive organizational changes in terms of new approaches to system usage and different IT governance mechanisms, as well as changes in the roles and responsibilities of employees and Information Systems users in particular.

This paper is organized as follows: section II will provide the definition of the notion of System of Systems (SoS) and System of Information Systems (SoIS) while linking both SoS and SoIS tp the concept of Digital Ecosystem. After that, in section III, the SoIS architectural model is presented and then discussed by defining the concept of SoIS. Then, section IV, present MEMORAe SoIS as an example following the architectural model of SoIS. Section V will present a discussion of the role of knowledge base in the SoIS. Finally, the paper conclude with section VI.

\section{INFORMATION SySTEM OF SySTEMS (SOIS) VERSUS DIGITAL ECOSYSTEM}

In this section will go through various definitions of System of Systems presented in the literature and summarize them. Then, discuss a specific type of SoS that focuses on Information Systems working together in the orchestration of System of Information Systems while linking both SoS and SoIS tp the concept of Digital Ecosystem.

The notion of System of Systems (SoS) can be viewed as an evolution of the standard notion of systems. Many definitions of a SoS exist. An aggregate of systems leads to the creation of new forms of systems which may be either described within the framework of composite systems, or demonstrate additional 
features which add complexity to the description and may be referred to as System of Systems.

We can summarize the definitions mentioned in [3] [4] [5] [6]. In the light of the definitions mentioned in the literature, the notion of System of Systems (SoS) can be described as the following; Systems of systems are large-scale integrated systems which are heterogeneous and independently operable on their own, but are networked together for a common goal. The goal, as mentioned before, may be cost, performance, robustness, etc. In other terms, A System of Systems is a super system comprised of other elements which themselves are independent complex operational systems and interact among themselves to achieve a common goal. Each element of a SoS achieves well-substantiated goals even if they are detached from the rest of the SoS.

The evolution of SoS and its ability to solve problems comes from these three major factors:

- Autonomy: the systems involved in SoS are at least partially autonomous

- Local views: no system has a full global view of the SoS.

- Decentralization: there is no designated single controlling system, but decision and information gathering is distributed.

Based on the work in [7] [8] it is possible o view the System of Systems as a Digital Ecosystem (DE), where different systems of the SoS can be considered as species in the DE. Also base on the model of DE presented in [7], one of the systems composing SoS can play the role of the leader system as seen in the example provided in a following section about MEMORAe SoIS. Furthermore, the resemblance between SoS and DE can be found in the description of DE by [9]. DE may be described as a network of niches, interconnected with or via the platform, suited to the co-evolution of stakeholders. Similarly, [6] defines SoS as a set of different elements connected or related so as to perform a unique function not performable by the elements alone. These elements (systems) are the network of niches in the DE as mentioned in the previous description.

Next, a special type of SoS called System of Information Systems is defined. This SoIS operates as a single entry point for several Information Systems granting the user access to information and resources produced from multiple Information Systems, and providing the ability to manage resources coming from those different systems to even create an added value not possible to maintain when those systems were operating separately. Similar to SoS, SoIS can be considered as Digital Ecosystems where Information Systems takes the role of species (or network of niches) in the DE model.

The notion of System of Information Systems is defined by [10] as "networks of agents interacting in a specific technology area under a particular institutional infrastructure for the purpose of creating, diffusing, and utilizing technology focused on knowledge, information, and competence flow." [11] describe SoIS as "the specific clusters of the firms, technologies, and industries involved in the generation and diffusion of new technologies and in the knowledge flow that takes place among them."

Based on the definitions provided the features of SoIS can be summarized as follows:

- SoIS addresses the impact of the interrelationships between different IS.

- SoIS is concerned with the flow of information and knowledge among different information systems.

- SoIS is responsible for generating information from the emergent IS.

- Information interoperability is a key issue when designing a SoIS.

\section{ARChitectural Model OF SyStem OF INFORMATION SYSTEM}

This part presents the generic architecture of the SoIS. As seen in (Fig. 1), the SoIS will aggregate information and resources from several Information Systems (System A, System B etc.). These systems are working separately. Each of which has its own services/functions and databases. The services/functions of these systems are denoted as solid rectangles inside the system (Service/Function 1A, Service/Function $1 \mathrm{~B}$ etc.). There is a difference here between services and functions. While some systems are openly providing an API for requesting their services, other systems are closed and operate as black boxes to the outer world and only provide functions invoked within the system itself. Information can be represented in different ways within different systems, thus, the SoIS might have trouble access information, unless the services of that system are available through an API. Therefore, there is a growing need for a solution to this interoperability issue. A solution to the problem of assuring interoperability within the SoIS is to control the communication medium among the systems. Two methods, outlined by [12], include:

- Creating a software model of each system, where the software model collects data from the system and generates the outputs.

- Creating a common language to describe data, where each system can represent its data such that other systems may interpret.

Due to overhead restrictions on a common language, it is not often that standard language for data interpretation is used throughout the SoIS. Therefore, creating individual software models for the various systems is the most widely used approach to ensure interoperability within a SoIS. This can explain the need for data wrapper as a mediator software between the SoIS and its component systems. The SoIS is represented as a group of systems connectors, services and databases. The services residing in the SoIS can either be utilization of existed services from the Information Systems comprising the SoIS, or emerging services created from the aggregation of different Information Systems.

The ideas present in this architecture of SoIS can be summarized in the following list:

- The user is provided with access to several Information Systems. 


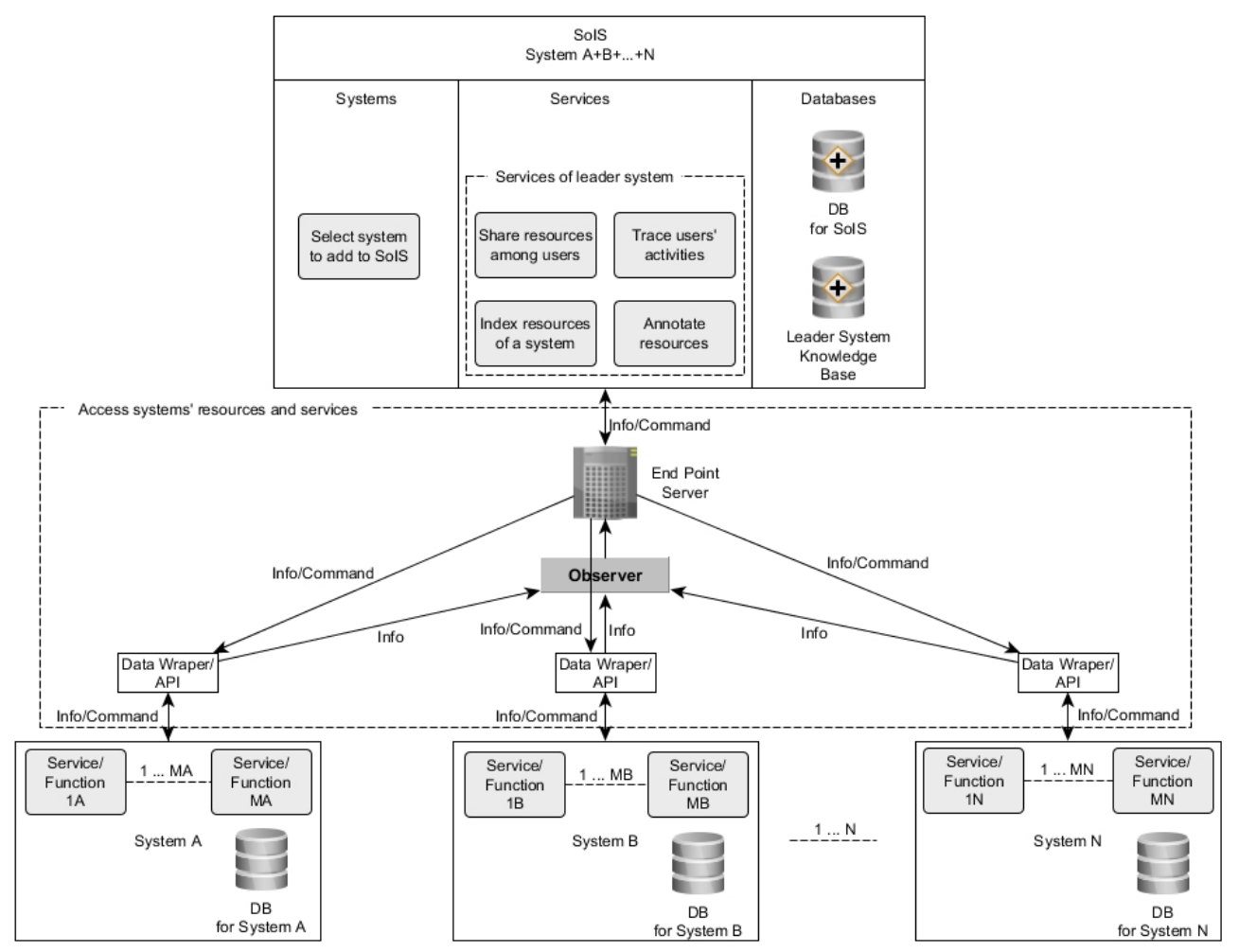

Fig. 1. Architectural model of the SoIS.

- The user can choose which Information System(s) he/she would like to connect to.

- After connecting to various systems of choice, the user can access resources and services in their respective environment.

- It is then possible to work with resources produced by different Information Systems from within the SoIS.

- The resources produced by different Information Systems are managed in the SoIS by the means of the services provided by the leader system.

- Using the services of the leader system as a way to manage knowledge with the SoIS will enable the user from indexing, sharing and tracing resources within the SoIS.

\section{MEMORAE SOIS}

This section proposes a prototype developed based on the architectural model presented earlier. As mentioned in the architecture of SoIS, leader system plays a leading role in the orchestration of SoIS as a knowledge base serving all other Information Systems. We propose MEMORAe system to play the role of the leader system in the SoIS. So, what is MEMORAe?

As defined by [13], MEMORAe approach is to manage heterogeneous information resources within organizations. The approach is comprised of a semantic model (called MEMORAe-core 2) and a web platform (called MEMORAe) which is based on the semantic model. The model and the platform make together a support to enhance the process of organizational learning.

The MEMORAe project uses the Semantic Web standards, therefore, the ontologies used in the system are written in OWL. Users registered in MEMORAe system can access one or more knowledge bases. When a base is chosen, a user can view a semantic map of concepts related to the selected base. Then, a user can create and share resources around the concepts of the map.

The user of MEMORAe web platform is able to create various resources within the platform. These resources include documents, notes, clusters of notes, weblinks, contacts, events, tours, and forums. The user is also able to index these resources using the semantic map visualized as concepts and instances in MEMORAe. Furthermore, MEMORAe gives users the ability to annotate these resources and share them within different sharing spaces, in addition to tracing users' activities within the platform. The limitation here is in the resources available inside MEMORAe. While the platform permits creating and managing various types of resources, all of them are native to the platform. Users might benefit more if the capabilities provided by MEMORAe approach (indexing, annotating, sharing, tracing the resources) is applicable to resources created by other Information Systems in the work scope of the user but outside the scope of MEMORAe. Such resources might be emails, wiki pages, social media entries, and others related to users' work. Hence, this paper proposes to develop a prototype based on the architecture of SoIS that puts MEMORAe approach as a leader system among other Information Systems in this Digital Ecosystem. That 


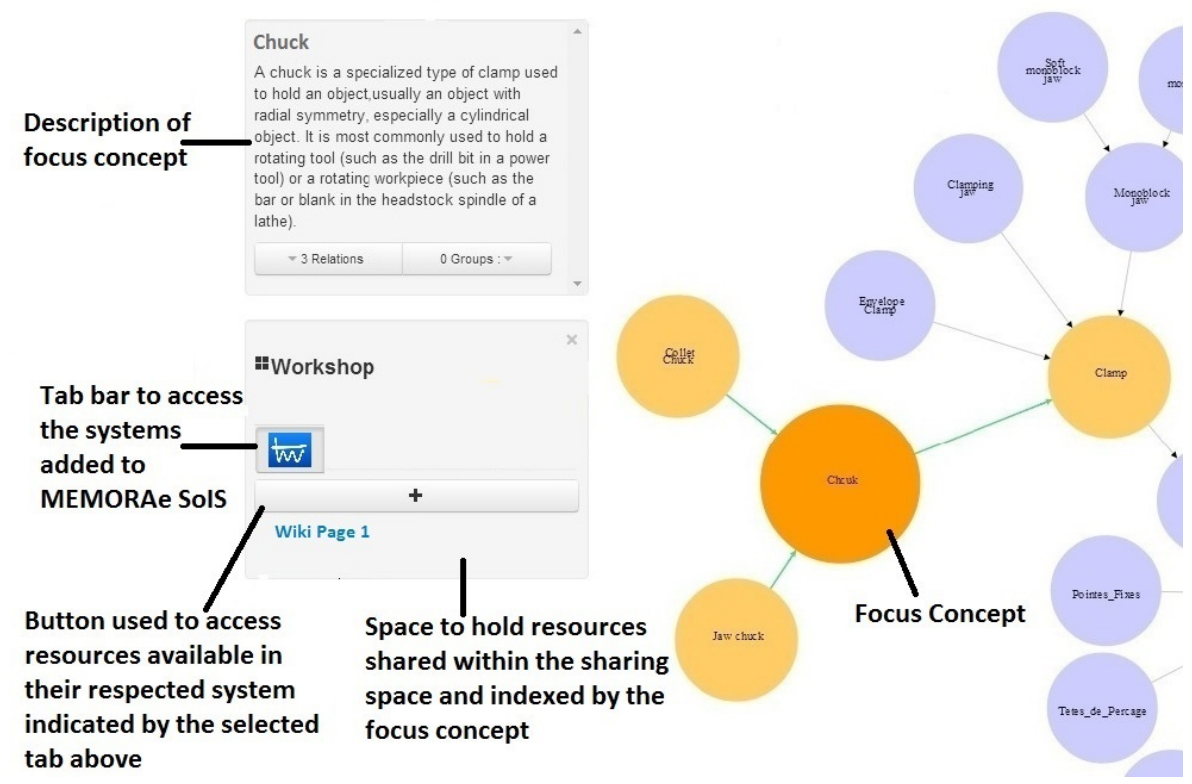

Fig. 2. MEMORAe SoIS with TiddlyWiki.

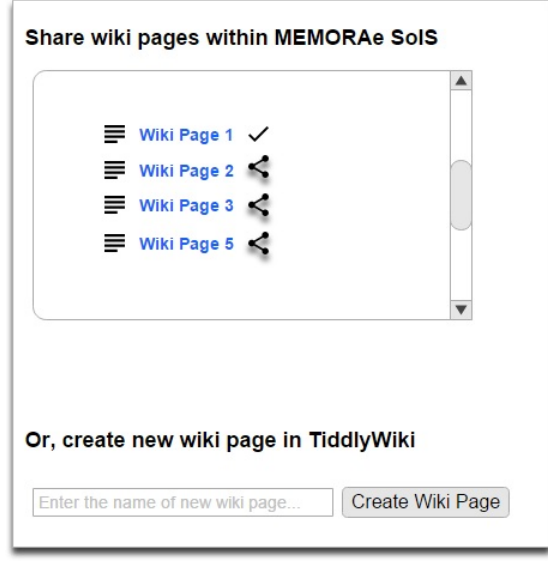

Fig. 3. Panel to access resources available in the Information System, or create new ones.

means taking MEMORAe web platform and evolve it into a MEMORAe SoIS. This can be developed following the SoIS architecture presented earlier with MEMORAe as a leader system and other Information Systems as composite systems of this SoIS. By doing so, users are given a wider work scope with the following features:

- Access resources coming from different Information Systems in a centralized location.

- Create resources using a dedicated Information System. These resources can be accessed either from the MEMORAe SoIS or the dedicated system where they are created.

- Organize resources around a semantic map.

- Share resources within different sharing spaces.

- Annotate resources in order to highlight certain ideas related to the resources.
- Record the users activities inside MEMORAe SoIS.

As an example, this paper will highlight the functionality of TiddlyWiki system and how it fits as a choice of the administrator to be added to MEMORAe SoIS. Technically, a TiddlyWiki is just an HTML page with a rather large JavaScript section that takes care of displaying all the contents, and provides the interactive tools for their manipulation. The actual text of the page is not immediately visible. It is stored in a set of invisible DIV elements, called tiddlers. The content of the DIV tags is wiki text, i.e. text with a simple markup language, similar to (old) emails. When the user clicks on a tiddler name to show its content, the JavaScript rendering machine translates the wiki text into HTML. The text may also contain macro fragments that trigger the actions of subroutines. Other tiddlers are interpreted as a stylesheet or a JavaScript plugin. When the user asks for a tiddler to be edited, this is replaced inline by a form, and the user is presented with the original text [14].

After the administrator chooses TiddlyWiki to be added to MEMORAe SoIS, all the users of the platform are able to access their TiddlyWiki resources, mostly wiki pages entries, from MEMORAe SoIS as seen in (Fig. 2). In this figure we can see the tab bar used to access different Information Systems. For this example it holds the tab for TiddlyWiki system. Previously, this tab bar in MEMORAe web platform only has native resources to choose from, while in MEMORAe SoIS the work scope is expanded to resources from other Information Systems such as TiddlyWiki. Moreover, for each tab in the tab bar there is a button with a "plus" sign. this button will allow the user to navigate through all the resources available in the dedicated Information System, which is TiddlyWiki in our example, and select from the list of resources which are going to be indexed by the semantic map of MEMORAe and shared by certain sharing spaces. It also allow the user to create new resources in their respected Information System. The panel to navigate through resources or create new ones is shown in (Fig. 3). The resources of 
the dedicated Information System is made available to the user by means of the data wrapper and the server/observer endpoint shown in the architecture in (Fig. 1).

\section{DISCUSSION}

This section will elaborate on the role of MEMORAe System as a knowledge base in the orchestration of SoIS, and the importance of the features it holds in providing the added value for the SoIS.

In the Example of SoIS presented earlier in section IV MEMORAe system can be viewed as a knowledge base that handles storing, organizing, and sharing of resources from different Information Systems. MEMORAe system aims, by its design, to facilitate knowledge sharing and capitalization within those different systems. All types of resources are indexed by a semantic map that represents the concepts of interest for the user [15]. furthermore, The semantic map defines a common reference shared between all users. The focus concept of the semantic map is a concept in which the user is interested in. The shared resources are indexed by the concepts of the semantic map. In this orchestration of SoIS, MEMORAe is introduced to new resources produced by Information Systems that reside outside its scope, hence the use of MEMORAe as it supports different types of social resources (wiki, forums, chat) and documentary resources (documents, images, web link). This constitutes an added value for the Information Systems comprising the SoIS, as data produced from thoes systems is capitalized as knowledge within MEMORAe.

User activity traces and recommendation system is another feature of MEMORAe that emphasize on the role of a knowledge base within a SoIS. MEMORAe systems owns a mechanism that models, records, and analyzes users traces. It allows evaluating competencies for recommending users with more expertise on certain subjects for either personal purpose or to an entire group of users [16]. Additionally to the previous point of MEMORAe capitalizing knowledge from different Information Systems, users' activities from those systems are also capitalized by using MEMORAe as a knowledge base in the SoIS.

\section{CONCLUSION}

The goal of this paper was to manage the resources obtained from different Information Systems and support decision making of users' competency within the environment of a System of Information Systems with simplicity and ease. The aim was focused towards investigating the knowledge base role in facilitating resources management in a System of Information Systems (SoIS), and providing an architectural model of SoIS to guide the migration for such complex system. To achieve this goal this paper undertakes a research to define the concept of System of Systems (SoS) and System of Information Systems (SoIS), then move based on the definitions to present an example of a SoIS composed of various Information Systems with MEMORAe approach in its core as a system to manage the resources produced by different systems comprising the SoIS. This paper found potentials in deploying MEMORAe approach to manage the resources produced by different systems in the SoIS. It was also clear that combining resources from various Information Systems and manage them within a knowledge base will result in an added value to users not present when those systems were operating separately.

The next step is to expand our work and introduce new Information Systems to the SoIS based on the example presented in this study and users needs. It should also be tested in a real world experiment. The SoIS should keep simple interface, with all the services as far from the user as a single click, to keep the users experience useful and friendly.

\section{ACKNOWLEDGMENT}

This project is done under ECOPACK project and funded by ANR-ASTRID programme.

\section{REFERENCES}

[1] H. An, "Research on information systems problems based on an ecological angle," in Challenges in Environmental Science and Computer Engineering (CESCE), 2010 International Conference on, vol. 1. IEEE, 2010, pp. 281-284.

[2] J. J. Simpson and C. H. Dagli, "System of systems: Power and paradox," in System of Systems Engineering, 2008. SoSE'08. IEEE International Conference on. IEEE, 2008, pp. 1-5.

[3] M. Jamshidi, System of systems engineering: innovations for the twentyfirst century. John Wiley \& Sons, 2011, vol. 58.

[4] P. G. Carlock and R. E. Fenton, "System of systems (sos) enterprise systems engineering for information-intensive organizations," Systems engineering, vol. 4, no. 4, pp. 242-261, 2001.

[5] W. H. Manthorpe, "The emerging joint system of systems: A systems engineering challenge and opportunity for apl," Johns Hopkins APL Technical Digest, vol. 17, no. 3, p. 305, 1996.

[6] E. Rechtin and M. W. Maier, The art of systems architecting. CRC Press, 2000.

[7] M. Saleh and M.-H. Abel, "Moving from digital ecosystem to system of information systems," in Computer Supported Cooperative Work in Design (CSCWD), 2016 IEEE 20th International Conference on, 2016.

[8] M. Saleh, M.-H. Abel, and V. Misseri, "Investigating the similarity between collaboration systems and digital ecosystems," in Computer Supported Cooperative Work in Design (CSCWD), 2015 IEEE 19th International Conference on. IEEE, 2015, pp. 30-35.

[9] A. Gawer and M. A. Cusumano, Platform leadership: How Intel, Microsoft, and Cisco drive industry innovation. Harvard Business School Press Boston, 2002.

[10] B. Carlsson and R. Stankiewicz, "On the nature, function and composition of technological systems," Journal of evolutionary economics, vol. 1, no. 2, pp. 93-118, 1991.

[11] S. Breschi and F. Malerba, Sectoral innovation systems: technological regimes, Schumpeterian dynamics and spatial boundaries. Centro studi sui processi di internazionalizzazione, Università commerciale'Luigi Bocconi', 1996.

[12] R. M. Bowen and F. Sahin, "A net-centric xml based system of systems architecture for human tracking," in System of Systems Engineering (SoSE), 2010 5th International Conference on. IEEE, 2010, pp. 1-6.

[13] C. M. Ala Atrash, Marie-Hélène Abel, "Supporting organizational learning with collaborative annotation," in 6th international conference on knowledge management and information sharing (KMIS), 2014, pp. 237- 244.

[14] F. Bagnoli, P. Jipsen, and A. Sterbini, "Tiddlywiki in science education," 2006.

[15] A. Atrash, M.-H. Abel, C. Moulin, N. Darène, F. Huet, and S. Bruaux, "Note-taking as a main feature in a social networking platform for small and medium sized enterprises," Computers in Human Behavior, 2015.

[16] N. Wang, M.-H. Abel, J.-P. Barthes, and E. Negre, "Mining user competency from semantic trace," in Computer Supported Cooperative Work in Design (CSCWD), 2015 IEEE 19th International Conference on. IEEE, 2015, pp. 48-53. 